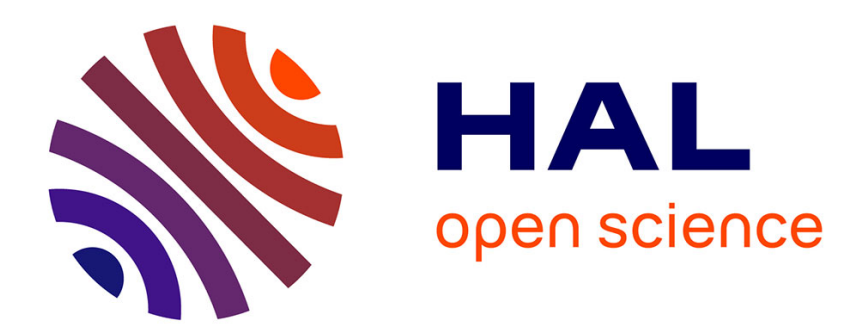

\title{
Le genre comme outil en didactique de la littérature
} Brigitte Louichon

\section{To cite this version:}

Brigitte Louichon. Le genre comme outil en didactique de la littérature. La lettre de l'AIRDF, 2018. hal-01765331

\section{HAL Id: hal-01765331 \\ https://hal.umontpellier.fr/hal-01765331}

Submitted on 12 Apr 2018

HAL is a multi-disciplinary open access archive for the deposit and dissemination of scientific research documents, whether they are published or not. The documents may come from teaching and research institutions in France or abroad, or from public or private research centers.
L'archive ouverte pluridisciplinaire HAL, est destinée au dépôt et à la diffusion de documents scientifiques de niveau recherche, publiés ou non, émanant des établissements d'enseignement et de recherche français ou étrangers, des laboratoires publics ou privés. 


\title{
Le genre comme outil en didactique de la littérature
}

\author{
Brigitte Louichon \\ Université de Montpellier \\ LIRDEF EA 3749
}

\section{De quelques constats}

En 2016, Françoise Cahen lançait une pétition dénonçant l'absence d'auteure au programme de littérature de Terminale L. En 2017, une nouvelle pétition s'insurge pour les mêmes raisons, relativement cette fois au programme de l'agrégation de lettres ${ }^{1}$.

Ces pétitions rendent visibles un constat opéré de longue date : les auteures sont très peu présentes dans les manuels ${ }^{2}$, les anthologies ${ }^{3}$, les programmes ${ }^{4}$ et les concours ${ }^{5}$, lors même que les lycéens de Terminale L sont surtout des lycéennes, que les étudiants de lettres sont surtout des étudiantes et que les professeurs de lettres sont surtout des professeures.

Mme de Lafayette sera au programme de Terminale cette année. C'est une forme de victoire et le mouvement doit s'accentuer. Mais peut-on s'en satisfaire ? Revendiquer la présence de femmes sur ces listes amène à rendre aussi visible leur faible nombre. Comme l'écrit Françoise Cahen : «il ne s'agit pas de demander la parité ». Ce que ces pétitions (et quels que soient les résultats obtenus) mettent en exergue, c'est qu'il y a quelques grandes auteures, écrivaines et qu'elles sont peu nombreuses. Ce sera sans doute une victoire que d'avoir une auteure de manière systématique à l'agrégation mais quelle problématique victoire ! Cela ressemble fort à ce que Martine Reid nomme la «valorisation paradoxale des écrits de femmes », c'est-à-dire « le fait de célébrer une femme auteur au détriment d'autres femmes auteurs de la même époque » $(2013,13)$.

La recherche n'ignore pas la dimension genrée de la littérature. Elle s'affirme dans une perspective essentiellement historique et sociologique. Le problème n'est plus celui de la définition d'une «écriture féminine » et l'on ne peut que s'étonner de lire ce qu'écrivait Béatrice Didier à propos des œuvres écrites par des femmes : «ce qui frappe [...] c'est un accent, la marque d'une différence qui rend habituellement reconnaissable un texte écrit par une femme » (Didier, 1981, 17). Or une œuvre n'est pas seulement une forme ${ }^{6}$, elle est aussi

\footnotetext{
${ }^{1} \mathrm{http}: / / \mathrm{fcahen}$. neowordpress.fr/

${ }^{2}$ https://m.centre-hubertine-auclert.fr/sites/default/files/fichiers/etude-2013-francais-cha-web_1.pdf

${ }^{3}$ Fraisse (1997)

${ }^{4}$ https://womenandfictionblog.wordpress.com/2016/06/18/autrices-concours-et-canon-1-les-chiffres/

${ }^{5}$ http://fcahen.neowordpress.fr/2017/03/25/sois-agregee-et-tais-toi/

${ }^{6}$ Contrairement à ce qu'affirme Jean-Yves Tadié, directeur de La Littérature française : dynamique \& histoire I et II (Gallimard), lorsqu'il est interrogé sur la faible place des femmes dans l'histoire littéraire qu'il a dirigée http://www.fabula.org/lht/7/tadie.html. En revanche, Barbey d'Aurévilly, l'un des pires contempteurs des écrivaines écrit aussi : "Quelqu'un sans savoir que les livres sont d'elles, les ouvrirait au hasard, y reconnaîtrait à toute ligne l'âme d'une femme, la pensée d'une femme [...] il y a un accent de pureté, de sincérité et de tendresse, d'amour pour tous les sacrifices, de respect pour tous les enthousiasmes, qui révèle bien toute la femme qu'elle fut ». (Barbey d'Aurévilly, 2006, 43). On le voit, la notion d'écriture féminine est à manier avec circonspection!
} 
une production inscrite dans un contexte et une époque donnés. En France, l'ouvrage de Christine Planté, La petite sœur de Balzac, paru en 1989, a ouvert de nouvelles perspectives (Reid, 2010 ; Del Lungo \& Louichon, 2010, 2013, 2017) qui mettent au jour des œuvres et des auteures mais s'interrogent surtout sur les modalités d'exclusion, de sélection et d'oubli (Del Lungo, 2017). Dans ces travaux, la question de l'histoire littéraire est centrale (Planté, 2003 ; Reid, 2011, 2016).

Pour faire (très) court, en France, l'histoire littéraire (en tant que discours constituant et méthode de description et de structuration des savoirs) est le socle de l'enseignement de la littérature et l'Histoire de la littérature française de Lanson (1895) «marque le début d'un savoir structuré [...] dont toutes les tentatives qui ont suivi sont restées, et restent encore aujourd'hui, généreusement tributaires » (Reid, 2016, 27). Or, du point de vue du genre, les histoires littéraires, de manières plus ou moins différenciées, à la suite de Lanson, donnent une place très faible aux femmes et les assignent à certains genres littéraires, nécessairement mineurs. Quelques noms d'écrivaines émergent : Pizan, Labé, Sévigné, Lafayette, Staël, Sand, Colette, Yourcenar, Duras, Ernaux... Nul n'ignore la fonction idéologique de l'histoire littéraire et le rôle premier qu'elle joue dans l'élaboration du canon.

C'est un fait. Avec lequel il faut faire. Avec lequel les enseignant.e.s de lettres doivent faire.

Mais l'enseignement de la littérature n'est pas leur seul apanage. Dès la maternelle, à l'école primaire, les œuvres littéraires sont présentes. Ce sont des œuvres de littérature jeunesse. La recherche dans ce domaine se développe aussi à partir de catégories genrées (Connan-Pintado C., Béhotéguy G., 2014). Mais, de manière assez systématique, les travaux s'intéressent peu à la production et aux auteur.e. $\mathrm{s}^{7}$. Ils se déplacent du côté du personnage, de la représentation du féminin et du masculin. La question est initialement posée en termes de «modèle » (Chombart de Lauwe, 1965 ; Cromer \& Turin, 1998) proposés par les fictions et à partir desquelles les jeunes lectrices et lecteurs se construisent de manière socialement différenciée. Les recherches plus récentes s'appuient plutôt sur la notion de «stéréotype » (Chabrol-Gagne, 2011) et de son corollaire, le « contre-stéréotype » (Ferrière \& Morin-Messabel, 2014).

L'étude de la littérature au prisme du genre conduit donc à une différenciation assez nette. Lorsque l'on s'intéresse à la littérature scolarisée dans le secondaire, la question du sexe de l'auteur.e est première. Lorsque l'on s'intéresse à la littérature scolarisée dans le primaire, la question de la représentation du personnage est prioritaire. Et ces recherches aboutissent aux constats que l'on sait: la littérature enseignée au secondaire est une littérature très majoritairement produite par les hommes et la littérature enseignée au primaire est une littérature dans lesquels les stéréotypes, même s'ils évoluent (Cromer, 2014) contribuent à des représentations toujours sexistes.

Par rapport à ces constats, la marge de manœuvre des enseignant.e.s est faible. Ils et elles ne peuvent réécrire l'histoire littéraire. Ils et elles ne peuvent échapper à la littérature pour la jeunesse telle qu'elle continue à s'écrire. Ils et elles sont contraint.e.s par les corpus.

\footnotetext{
${ }^{7}$ Dans une étude relative à la liste de référence du MEN de 2002, Cromer dénombre 80\% d'auteurs hommes. Lydie Laroque à partir de la liste 2013 dénombre 29\% d'auteures parmi les titres nouveaux (Manuelian, Magnan-Rahimi et Laroque 2014). Il est pourtant significatif que l'essentiel de leur propos se concentre sur les personnages
} 


\section{Le genre comme outil didactique}

Dès lors, le genre doit être pensé comme un outil pour enseigner. C'est-à-dire un outil didactique.

Il s'agit d'abord de faire en sorte que les enseignant.e.s prennent conscience et connaissance de la manière - très rapidement esquissée ici - dont les corpus et les œuvres singulières peuvent être pensés dès lors que l'on adopte un point de vue genré. Le fait que la littérature enseignée soit produite par des hommes et qu'elle représente le masculin et le féminin de manière stéréotypée n'est perceptible que si l'on veut bien d'abord y prêter attention et ensuite considérer que cela a un impact sur le devenir des élèves. Autrement dit, le genre doit être un outil en formation, non seulement transversale, comme c'est souvent le cas dans les masters MEEF, mais aussi spécifiquement en formation disciplinaire, à l'université, et dans les ESPE, en formation didactique (Dias-Chiaruttini, 2015), et pas seulement par le biais de quelques mémoires ne formant - certes en général efficacement- que quelques professeur.e.s stagiaires. Pour ne prendre qu'un exemple, le travail sur le roman devrait nécessairement poser la question de la manière dont l'histoire du genre (littéraire) est travaillée par la question du masculin/féminin. Pour le primaire, il conviendrait de rendre les enseignant.e.s plus attentif.ve.s au simple fait qu'un livre, qu'un texte a un.e auteur.e ! Ils et elles ignorent en effet parfois le nom de l'auteur.e (Louichon, 2008, 57).

Une fois que les enseignant.e.s sont rendus conscients de ces phénomènes invisibles ou plus exactement naturalisés, quelles sont les pistes d'action?

Il convient de les envisager dans une double dimension : internaliste et matérialiste (Sofio, s. d.). La dimension internaliste s'intéresse au discours de l'œuvre et plus particulièrement aux personnages dans le cadre des cuvres de fiction. La seconde s'intéresse aux conditions de production, aux auteur.e.s. Les deux approches permettent de mettre au jour et de questionner les représentations du masculin et du féminin et de leurs relations, d'interroger le système de valeurs par lequel elles sont instituées et, qu'en retour, elles instituent. Ces deux approches peuvent se développer aux deux niveaux d'enseignement (primaire et secondaire).

\section{L'approche matérialiste}

Bien évidemment il convient de donner toute la (petite) place qui revient aux auteures. De même que les programmes de Terminale L ou d'agrégation doivent s'ouvrir à des œuvres de femmes, les corpus scolaires doivent aussi prendre cette voie. De même que l'enseignant.e cherche à équilibrer les œuvres en fonction des périodes, des genres (littéraires), des formes, il ou elle doit avoir à l'esprit ce paramètre dès lors qu'il est compris comme un paramètre didactique.

Cependant, nous l'avons dit, l'équilibre est impossible à obtenir. Dans cette logique, c'est à un questionnement que l'on doit conduire les élèves. L'analyse de manuels, souvent utilisée en recherche, est rarement pensée comme une modalité pédagogique (voir néanmoins Mercier, 2016). Elle permet pourtant de commencer par énoncer un fait qui doit l'être, sauf à ce qu'opère un phénomène de naturalisation. Ce fait peut alors être mis en discussion, c'est-àdire soumis à explication (et non argumentation). On peut par exemple, mais il y a sans doute plusieurs manières de faire, proposer un florilège de propos misogynes sur les auteures, qui rend lisibles les systèmes de valeurs en jeu, leur historicité et leur caducité. 
La littérature contemporaine offre indéniablement des opportunités bien supérieures à ce que l'histoire littéraire nous a légué ${ }^{8}$. Les programmes du lycée, qui posent pour chaque axe un empan temporel allant jusque «à nos jours », permettent de fait le recours à la littérature contemporaine. Mais nécessairement marginalement. D'autre part, les lectures cursives, complémentaires ou d'été proposées aux élèves intègrent en général une part importante de littérature contemporaine, et, ce faisant, de littérature écrite par des femmes. Pour autant, cette pratique met en exergue le même système de valeurs: aux grands auteurs, la lecture analytique, les listes de bac; aux femmes (et au contemporain, et au francophone...) une lecture plus légère, facile, non ou peu évaluée.

Toujours dans la perspective matérialiste, il paraît intéressant d'avoir en tête le paramètre didactique du genre lorsque l'on a peut mettre en relation des élèves et des auteur.e.s vivant.e.s et sans doute est-ce là une manière efficace de répondre à la question des modèles et des représentations. Qu'il s'agisse de l'accueil d'un.e auteur.e en résidence, d'ateliers d'écritures ou plus simplement de rencontres à l'occasion d'un projet, d'un salon du livre, il convient de se demander aussi si l'on préfèrera faire entrer une femme ou un homme dans la classe $^{9}$, si les élèves de maternelle, d'élémentaire, de collège, de lycée, les étudiant.e.s, les fonctionnaires stagiaires, les professeur.e.s en formation continue, garderont en mémoire un travail, une rencontre, un dialogue avec une femme ou un homme ${ }^{10}$. Ces activités pédagogiques parfois discutées tant par les didacticiens (Guernier, 2007) que par les auteur.e.s (Dayre, 1997) pourraient ainsi trouver une finalité, qu'à ma connaissance, on a peu mise en exergue. Nul doute que la rencontre de lycéens avec Maylis de Kérangal ${ }^{11}$, auteure distinguée (au sens critique du terme), construit des représentations et des modèles susceptibles de contrebalancer efficacement celles que l'enseignement des lettres (patrimoniales) induit.

\section{L'approche internaliste}

Il s'agit tout simplement de la lecture et de l'étude des œuvres, celles-ci constituant l'essentiel de l'enseignant.e de littérature à l'école, au collège, au lycée.

Le personnage constitue une des entrées premières qu'il s'agisse de lecture (Jouve, 1992), d'analyse littéraire (Hamon, 1972, Glaudes \& Reuter, 1998) ou de didactique (Tauveron, 1995). Les catégories auxquelles il convient de recourir (désignation, caractérisation, distinction, hiérarchisation, fonctionnalité, systèmes des personnages, instance narrative...) et l'attention aux discours du personnage, aux discours sur le personnage, et au discours du

\footnotetext{
${ }^{8}$ Encore qu'il faille demeurer vigilant ! Une double page récente du Monde (18/08/17, p. 16-17) est consacrée à la rentrée littéraire. Elle est due à Raphaëlle Leyris qui signe les quatre articles qui la composent. Le premier est introductif et ne cite pas d'auteur.e.s. Le second est consacré au «triomphe de l'exofiction ». Pour soutenir son propos, la journaliste s'appuie sur la production effective. Elle cite alors 16 romanciers et 16 romancières. Le troisième article concerne les «têtes d'affiche », les «stars des maisons d'édition ». Là, 12 romancières et 23 romanciers sont cités. Enfin, le dernier article porte sur les « richesses étrangères », les « nombreux écrivains de tout premier plan », il recense 18 romanciers et 4 romancières. On observe ainsi que dès que la valeur (financière et/ou artistique) est en jeu, le masculin l'emporte encore sur le féminin. Le seul critère quantitatif (la production) ne suffit pas à appréhender la question, mieux vaut retenir celui de la distinction (Louichon, 2013).

${ }^{9} \mathrm{Cf}$ à titre d'exemple l'appel de la Région Occitanie de l'année 2016-2017 qui justement portait sur « une chambre à soi : à elle, à lui » http://www.lr21.fr/actualites/appel-projet-auteurs-au-lycee-2016-2017.html

${ }^{10}$ Il convient bien sûr de viser ici la parité. Il est tout aussi important que la littérature ne soit pas l'apanage des femmes lors même que l'on sait que la lecture comme pratique culturelle est plus féminine que masculine.

${ }^{11} \mathrm{Cf}$ https://pbellevue.libreedu.ovh/productions/wpcdi/index.php/2017/05/01/rencontre-avec-maylis-de-kerangal/
} 
roman sont à même de permettre la mise au jour des stéréotypes et des valeurs, y compris du point de vue du genre.

Les travaux plus récents sur «l'empathie fictionnelle» dans le champ de la didactique, posée comme une compétence de lecture et visant le lecteur à mieux comprendre les états mentaux des personnages sont aussi fort utiles. Ainsi Véronique Larrivé propose-t-elle un dispositif didactique qu'elle nomme «journal intime du personnage » grâce auquel les élèves sont amenés à produire une écriture en «je » pour comprendre les motivations du personnage. Il s'agit de s'interroger sur les pensées et les sentiments des personnages en adoptant leur point de vue (Larrivé, 2015), qui, nécessairement est un point de vue genré ${ }^{12}$. Dans le travail mené autour de La Belle et la bête (Larrivé, 2013), l'écart est ainsi spectaculaire entre des productions de type gnomique à la troisième personne et la production d'un discours en je du personnage principal qui engage des propos sur l'amour et la liberté à hauteur de sujet (genré).

Mais le genre est aussi une catégorie très prégnante chez les éditeurs jeunesse. Les ouvrages publiés le sont souvent à destination des garçons ou des filles. Par-delà les stratégies paratextuelles ( $4^{\text {ème }}$ de couverture, collections, images, couleurs...), on peut aussi parler de stratégies narratives, de stéréotypisation narrative. A travers ce prisme, B. Ferrier (2016) étudie les «stratégies de girlisation de l'Histoire dans les fictions pour la jeunesse », en dégageant six stratégies: la personnification, la déshistoricisation, l'embellissement, la scénarisation, la conscientisation et l'éditorialisation. Je fais l'hypothèse que cette notion de stéréotypisation narrative pourrait être utilisée à des fins didactiques, afin de rendre conscients les élèves (par une réception outillée et éventuellement par des exercices de réécriture) de la manière dont se fabriquent les catégories naturalisées : les livres pour les filles et les livres pour les garçons.

On l'aura compris, penser le genre comme outil didactique consiste simplement à ce les œuvres, les outils d'analyse et les modalités soient pensés en tant qu'ils peuvent orienter un enseignement dont la finalité soit égalitaire et émancipatrice.

\section{Bibliographie :}

Barbey d'Aurévilly (2006). Les Bas-bleus in Auraix-Jonchière P. , Glaudes P., Mayaux C (éd). Euvres critiques, tome 2. Paris : Les Belles Lettres.

Chabrol-Gagne, Nelly (2011). Filles d'album. Les représentations du féminin dans l'album. Le Puy-en-Velay : L'Atelier du poisson soluble.

Chombart de Lauwe, Marie-José (1965). Convergences et divergences des modèles d'enfants dans les manuels scolaires et la littérature enfantine. Psychologie française, n³, p. 236-244.

Connan-Pintado, C. \& Béhotéguy, G. (dir.) (2014). Être une fille, un garçon dans la littérature pour la jeunesse. France 1945-2012. Bordeaux : Presses universitaires de Bordeaux. Coll. Etudes sur le livre de jeunesse.

\footnotetext{
${ }^{12}$ Ce qui implique que les personnages choisis soient des hommes et des femmes.
} 
Cromer S. \& Turin A. (1998). Quels modèles pour les filles? Association « du côté des filles », 1998.

Cromer S. (2014). La littérature de jeunesse mise à l'épreuve du genre. In Connan-Pintado, C. \& Béhotéguy, G. (dir.) (2014). Être une fille, un garçon dans la littérature pour la jeunesse. France 1945-2012. Bordeaux : Presses universitaires de Bordeaux. Coll. Etudes sur le livre de jeunesse, p. 55-66.

Dayre V. (1997). Le Jour où on a mangé l'écrivain. Paris : L'école des loisirs. Coll. medium.

Del Lungo A. (2017). Les raisons d'un oubli. Quelques remarques sur l'exclusion des femmes du canon littéraire au tournant du XXe siècle. In Del Lungo A. \& Louichon B. (dir.). La Littérature en bas-bleus III. Romancières en France de 1870 à 1914. Paris : Classiques Garnier, p. 24-36.

Del Lungo A. \& Louichon B. (dir.) (2010), La Littérature en bas-bleus. Romancières sous la Restauration et la monarchie de Juillet. Paris : Classiques Garnier.

Del Lungo A. et Louichon B. (dir.) (2013). La Littérature en bas-bleus. T.II. Romancières 1848-1870. Paris : Classiques Garnier.

Dias-Chiaruttini A. (2015). Réception des stéréotypes genrés véhiculés par la littérature de jeunesse dans des espaces institutionnels contrastés. Repères, 51, p. 35-54.

Didier B. (1981). L'Ecriture-femme. Paris : PUF.

Ferrier B. (2016). La Fiction historique a-t-elle un genre ? Six stratégies de girlisation de l'Histoire dans les fictions pour la jeunesse. In Louichon B. \& Brehm S. Fictions historiques pour la jeunesse en France et au Québec. Bordeaux: Presses Universitaires de Bordeaux. Coll. Etudes sur le Livre Jeunesse, 297-315.

Ferrière S. \& Morin-Messabel C. (2014). Contre-stéréotypes et développement de l'identité de genre. In Connan-Pintado, C. \& Béhotéguy, G. (dir.). Être une fille, un garçon dans la littérature pour la jeunesse. France 1945-2012. Bordeaux : Presses universitaires de Bordeaux. Coll. Etudes sur le livre de jeunesse, p. 225-238.

Fraisse E. (1997). Les Anthologies en France. Paris : P.U.F. Coll. Ecritures.

Guernier M.-C. (2007). Eécrivain - élève - professeur, une rencontre problématique. In Bayard J.-L. \& Mercier-Faivre A.-M. Vous avez dit contemporain ? Enseigner les écritures d'aujourd'hui, Publications de l'Université de Saint-Etienne, p. 101-108.

Glaudes P., Reuter Y. (1998). Le Personnage. Paris : PUF. Coll. Que sais-je ?

Hamon P. (1972). Pour un statut sémiologique du personnage. Littérature, Vol. 6, n², p. 86110.

Jouve V. (1992). L'Effet personnage dans le roman. Paris : PUF.

Larrivé V. (2013). La Barbe bleue et « l'effet personnage »-En CM2 et 6e, des écritures en je pour réactualiser la lecture de la Barbe bleue. Ahr S. et Denizot N., Les Patrimoines littéraires : usages et enjeux. Namur : CEDOCEF. Coll. Diptyque, p. 119-136.

Larrivé V. (2015). Empathie fictionnelle et écriture en « je » fictif. Repères, 51, p. 157-176. 
Louichon B. (2008). Enquête sur le rapport des enseignants aux programmes de 2002 et les pratiques d'enseignement de la littérature au cycle 3. Repères, 37, p. 51-68.

Louichon B. (2013). Les romans en 1802. Romantisme, 160, p. 15-26.

Manuelian M., Magnan-Rahimi N. et Laroque N. (2016). La littérature pour la jeunesse et le genre : un corpus face à ses contradictions. Le Français aujourd'hui, 193, 45-60.

Mercier C. (2016). Des élèves concepteurs de pages de manuels. Recherches, 65, 129-146.

Planté, C. (1989). La petite sæur de Balzac. Paris : Seuil.

Planté, C. (2003). La place des femmes dans l'histoire littéraire : annexe, ou point de départ d'une relecture critique ?. Revue d'histoire littéraire de la France, vol. 103, (3),p. 655-668.

Reid M. (2010). Des Femmes en littérature. Paris : Belin.

Reid M. (dir.) (2011). Les Femmes dans la critique et l'histoire littéraires. Paris : Honoré Champion.

Reid M. (2013). Les Bas-bleus du Second Empire. In A. Del Lungo et B. Louichon (dir.), La Littérature en bas-bleus. T.II. Romancières 1848-1870, Paris : Classiques Garnier, p. 7-23.

Reid, M. (2016). L’histoire littéraire au prisme du « genre ». Le Français aujourd'hui, n 193, p. 25-32.

Sofio S. (s.d.). Genre (gender). In Glinoer A. \& Saint-Amand D. (dir.).Le lexique socius. URL : http://ressources-socius.info/index.php/lexique/21-lexique/65-genre-gender, page consultée le 28 aopt 2017.

Tauveron, C. (1995). Le Personnage. Une clef pour la didactique du récit à l'école élémentaire. Neuchâtel : Delachaux \& Niestlé. Coll. Techniques et méthodes pédagogiques. 